\title{
Relationship between interferon regulatory factor 4 genetic polymorphisms, measures of sun sensitivity and risk for non-Hodgkin lymphoma
}

\author{
Allison H. Gathany - Patricia Hartge $\cdot$ Scott Davis · James R. Cerhan • \\ Richard K. Severson - Wendy Cozen · Nathaniel Rothman · Stephen J. Chanock • \\ Sophia S. Wang
}

Received: 18 December 2008/Accepted: 7 April 2009/Published online: 28 April 2009

(C) The Author(s) 2009. This article is published with open access at Springerlink.com

\begin{abstract}
Objective Sun exposure and sensitivity, including pigmentation, are associated with risk for non-Hodgkin lymphoma (NHL). One variant in the immune regulatory factor 4 (IRF4) gene (rs12203592) is associated with pigmentation, and a different IRF4 variant (rs12211228) is associated with NHL risk. We evaluated the
\end{abstract}

Electronic supplementary material The online version of this article (doi:10.1007/s10552-009-9348-5) contains supplementary material, which is available to authorized users.

A. H. Gathany · P. Hartge · N. Rothman · S. J. Chanock Division of Cancer Epidemiology and Genetics, National Cancer Institute, NIH, DHHS, 6120 Executive Blvd., Rockville, MD 20852, USA

A. H. Gathany

Yale School of Public Heath, New Haven, CT, USA

S. Davis

Fred Hutchinson Cancer Research Center and University of Washington, Seattle, WA, USA

\section{J. R. Cerhan}

Mayo Clinic College of Medicine, Rochester, MN, USA

R. K. Severson

Karmanos Cancer Institute and Department of Family Medicine, Wayne State University, Detroit, MI, USA

W. Cozen

University of Southern California, Los Angeles, CA, USA

\section{S. S. Wang ( $\square)$}

Division of Cancer Epidemiology and Genetics, National Cancer Institute, NIH, DHHS, 6120 Executive Blvd., EPS 7070, Rockville, MD 20852, USA

e-mail: wangso@mail.nih.gov independent roles of these IRF4 polymorphisms and sun sensitivity in mediating NHL risk and explored whether they are confounded or modified by each other.

Methods Genotyping of tag single nucleotide polymorphisms (SNPs) in the IRF4 gene was conducted in 990 NHL cases and 828 controls from a multi-center US study. Measures of sun sensitivity and exposure were ascertained from computer-assisted personal interviews. We used logistic regression to compute odds ratios (OR) and $95 \%$ confidence intervals $(\mathrm{CI})$ for NHL in relation to sun exposures, sun exposures in relation to IRF4 genotypes, and NHL in relation to sun exposures. We further assessed the effects of sun exposures in relation to IRF4 genotypes.

Results As previously reported, we found significant associations between IRF4 rs12211228 and NHL and between hair and eye color and NHL. The IRF4 rs12203592 polymorphism (CT/TT genotype) was statistically significantly associated with eye color and particularly with hair color $\left(\mathrm{OR}_{\text {Light }}\right.$ Blonde $=0.24, \quad 95 \%$ $\mathrm{CI}=0.11-0.50$, overall Chi square $p=0.0002$ ). Analysis of joint effects between eye and hair color with the IRF4 rs12203592 SNP did not reveal statistically significant $p$-interactions although NHL risk did decline with lighter hair color and presence of the variant IRF4 rs12203592 allele, compared to those without a variant allele and with black/brown hair color.

Conclusions Our data do not statistically support a joint effect between IRF4 and sun sensitivity in mediating risk for NHL. Further evaluation of joint effects in other and larger populations is warranted.

Keywords Non-Hodgkin lymphoma .

Interferon regulatory factor-4 .

Single nucleotide polymorphism $\cdot$ Sunlight $\cdot$ Pigmentation 


$\begin{array}{ll}\text { Abbreviations } \\ \text { CAPI } & \begin{array}{l}\text { Computer-assisted personal interview } \\ \text { CI }\end{array} \\ \text { CLL/SLL } & \begin{array}{l}\text { Confidence interval lymphocytic leukemia/small } \\ \text { lymphocytic lymphoma }\end{array} \\ \text { DLBCL } & \begin{array}{l}\text { Diffuse large B-cell lymphoma } \\ \text { HHHQ }\end{array} \\ \text { Health habits and history questionnaire } \\ \text { HWE } & \text { Hardy-Weinberg equilibrium } \\ \text { ICD-O } & \begin{array}{l}\text { International classification of diseases for } \\ \text { oncology }\end{array} \\ \text { MAF } & \text { Minor allele frequency } \\ \text { NA } & \text { Not applicable } \\ \text { NCI } & \text { National cancer institute } \\ \text { NHANES } & \text { National health and nutrition examination } \\ & \text { survey } \\ \text { NHL } & \text { Non-Hodgkin lymphoma } \\ \text { OR } & \text { Odds ratio } \\ \text { SEER } & \text { Surveillance, epidemiology, and end results } \\ \text { SNP } & \text { Single nucleotide polymorphism } \\ \text { WT } & \text { Wild-type }\end{array}$

\section{Introduction}

Non-Hodgkin lymphomas (NHL) represent a heterogeneous group of cancers arising from lymphocytes for which the etiology remains largely unclear. While the major known risk factor for developing NHL is severe immunodeficiency [1], the increased NHL risk among those with a family history of hematopoietic disease [2-5] suggests an important role for genetic susceptibility in NHL etiology. Investigations of germline genetic variation in large epidemiologic case-control studies have identified potentially relevant loci that may play a role in modulating risk for NHL $[6,7]$.

A recent pooled analysis of three large population-based NHL case-control studies that included the present study found a significant association between a polymorphism in the IRF4 gene, rs12211228 and NHL risk [8]. The IRF4 gene (also known as $M U M 1$ ) encodes a protein that is a member of the interferon regulatory factor family [9-12]. IRF4 is a B-cell proliferation and differentiation protein essential for class switch recognition and antibody maturation $[13,14]$ and is often found abnormally expressed in B cell lymphomas [15]. In addition to serving as a marker for lymphoid tumors such as NHL, IRF4 has also been reported to act as a potential marker for melanoma [16].

A recent large genome-wide association study demonstrated a convincing association between a different polymorphism of the IRF4 gene, rs12203592, and pigmentation phenotypes, most notably hair color [17]. This IRF4 SNP was also shown to be associated with skin color, eye color, and a measure of skin tanning response to sunlight. This report was the first to suggest a link between a particular IRF4 locus and human pigmentation, though the mechanism for the involvement of this immune regulatory gene in determining hair color and other pigmentation phenotypes remains unclear.

Interestingly, sun sensitivity and sun exposure have previously been linked with NHL. Hartge et al. [18] previously showed a gradient of decreasing NHL risk with increasingly light eye color, one pigmentation phenotype believed to act as a marker of increased sun sensitivity. This relationship between risk for NHL and sun sensitivity and exposure is further supported by recent work from the International Lymphoma Epidemiology Consortium (InterLymph)[19], where a pooled analysis of ten case-control studies of NHL showed a significant decrease in NHL risk with increasing recreational sun exposure.

In order to clarify the roles of these two IRF4 polymorphisms and sun sensitivity in mediating NHL risk and to determine if their respective associations with NHL are confounded or modified by each other, we used a large US multi-center case-control study of NHL to evaluate the relationship between IRF4 polymorphisms and markers of sun sensitivity and sun exposure and their roles in determining risk for non-Hodgkin lymphoma.

\section{Materials and methods}

\section{Study population}

The study population has been described previously in detail [3]. Briefly, we included 1,321 newly diagnosed nonHodgkin lymphoma cases identified in four Surveillance, Epidemiology, and End Results (SEER) registries (Iowa; Detroit, MI; Los Angeles, CA; and Seattle, WA) between 1 July 1998 and 30 June 2000. Subjects were between the ages of 20 and 74 and had no evidence of HIV infection. A total of 1,057 population controls were identified by random digit dialing (under 65 years old) and from Medicare eligibility files (65 years and older). Overall participation rates were $76 \%$ in cases and $52 \%$ in controls; overall response rates were 59 and $44 \%$, respectively. Written informed consent was obtained from each participant before interview. This study was approved by the institutional review boards at the NIH and at each participating SEER site (Iowa, Detroit, LA, and Seattle).

All study participants were asked to provide a venous blood or mouthwash buccal cell sample. We obtained blood samples from 1,172 (89\%) cases (773 blood, 399 buccal) and 982 (93\%) controls (668 blood, 314 buccal). Genotype frequencies were equivalent for individuals who provided blood compared with buccal cells. 
Histopathology

Each SEER registry provided non-Hodgkin lymphoma pathology and subtype information derived from abstracted reports by the local diagnosing pathologist. All cases were histologically confirmed and coded according to the International Classification of Diseases for Oncology, 2nd Edition (ICD-O-2) [20] and updated to the World Health Organization classification/ICD-O-3 [21].

\section{Questionnaire data}

The study used a split-sample design to investigate multiple etiologic risk factors in detail without overburdening the participants. A core set of questions was given to all respondents, and the remainder of questions were given to participants in either Group A (all African-American participants and $50 \%$ of non-African-American participants) or Group B (50\% of non-African-American participants). Prior to the in-person interview, participants were mailed a form for listing residential and job history and either a family and medical history questionnaire (Group A) or a diet and lifestyle questionnaire (Group B). During the home visit, a trained interviewer administered a computerassisted personal interview (CAPI) that included core questions on demographics, height and weight, occupational history, pesticide exposure, hair color, and hair dye use. The Group A CAPI also included an extended medical history and use of illicit drugs, while the Group B CAPI included an abbreviated medical history, cell phone use, allergies, hobbies, eye color, skin complexion, and sun sensitivity/exposure.

Sun sensitivity and exposure. During the interview, we asked participants to estimate how many hours they spent in the sun during the summer in the middle of the day (10:00 AM-4:00 PM). We asked separately for weekdays and weekend days and separately for specific periods of their lives including teenage years, twenties, thirties, and the most recent decade. In the analysis, we estimated typical weekly exposure to strong sunlight as a weighted average of weekend and weekday values. We also asked about the use of sun lamps or tanning booths, the typical number of months per year they had a tan, pigmentation characteristics including eye color and skin complexion, and some common measures of skin response to sunlight, including sun rashes and typical reaction to first hour of sun with no tan and no sunburn.

\section{DNA extraction and genotyping}

Study participants who did not provide a biologic specimen, did not have sufficient material for DNA extraction or sufficient DNA for genotyping, or whose genotyped sex was discordant from the questionnaire data were excluded from this analysis. As previously described [22], DNA was extracted from blood clots or buffy coats (BBI Biotech, Gaithersburg, MD) using Puregene Autopure DNA extraction kits (Gentra Systems, Minneapolis, MN). DNA was extracted from buccal cell samples by phenol-chloroform extraction methods [23].

We selected 15 tag single nucleotide polymorphisms (SNPs) in the IRF4 gene as previously described [8]; these included the two a priori SNPs of interest-rs12211228 and rs12203592. Genotyping was conducted at the National Cancer Institute Core Genotyping Facility (Advanced Technology Center, Gaithersburg, MD) using a custom-designed GoldenGate assay (Illumina, www. illumina.com). Sequence data and assay conditions are provided at http://snp500cancer.nci.nih.gov [24]. SNP completion rates were $>95 \%$ for the 15 IRF4 SNPs. Forty replicate samples from two blood donors each and duplicate samples from 100 participants processed in an identical fashion were interspersed for all assays and blinded from the laboratory. The 15 IRF4 SNPs were $>95 \%$ concordant in the quality control samples. We excluded samples with a low completion rate $(<90 \% ; 11$ cases, 6 controls). Hardy-Weinberg Equilibrium (HWE) was observed in the control group for all IRF4 SNPs (assessed separately for non-Hispanic Caucasians and blacks).

The final analytic population consisted of 990 cases and 828 controls.

\section{Statistical analysis}

IRF4 and NHL. We calculated odds ratios (OR) and 95\% confidence intervals $(95 \% \mathrm{CI})$ as an estimate of relative risk for non-Hodgkin lymphoma outcomes using dichotomous (overall NHL) and polytomous (NHL subtypes) unconditional logistic regression models with the homozygous wild-type genotype as the referent group. We conducted stratified analyses by age ( $<60$ and $\geq 60$ years), sex (male and female), and race (non-Hispanic Caucasians and blacks). Finding no significant differences in the risk estimates by each of these three strata, we pooled the results and adjusted for the study design variables: age $(<50,50-59,60-69,70+)$, sex, race/ethnicity (non-Hispanic Caucasian, black, other), and study site (Iowa, Los Angeles, Seattle, Detroit). We calculated the $p$ for trend based on the three-level ordinal variable $(0,1,2)$ of homozygote wild-type, heterozygote, and homozygote variant. In addition to the individual risk estimates for each genotype, we evaluated the dominant model with homozygote wild-type as the referent group for comparison with heterozygotes and homozygote variants combined.

Sun sensitivity and NHL. In order to assess the association between the ordinal pigmentation and sun exposure 
variables and NHL, we calculated the OR and 95\% CI using dichotomous and polytomous unconditional logistic regression models for NHL overall and NHL subtypes, respectively. We also calculated the $p$ for trend value for linear trend in regression based on the categorical variables $(0,1,2$, etc.) for each level of exposure (e.g., for eye color: dark brown (0), light brown [1], hazel (2), blue (3), green/ blue-green (4)). For eye and hair color, skin complexion, reaction to first sun of the season, and hours in the mid-day sun in the last 10 years, the category corresponding to the lowest level of sun sensitivity or exposure was used as the referent category. All analyses were conducted both crude and adjusted for the study design variables age, race/ethnicity, sex, and study site. We note that we also included adjustment for education as a surrogate for SES; though education was slightly associated with sunlight exposure, additional adjustment for education did not appreciably alter the risk estimates for NHL $(<10 \%)$ and we therefore retained the most parsimonious model in our final model which excluded education. Secondary analyses restricted to subjects with genotype data and non-Hispanic Caucasian subjects with genotype data were also performed to assess consistency across population subgroups (adjusted for age, sex, and study site).

IRF4 and sun sensitivity. Among controls, we used logistic regression adjusted for age, sex, and study site to model the association between pigmentation or sun exposure and IRF4 genotypes. For each exposure category, the OR and $95 \%$ CI were calculated for the heterozygotes and homozygous variants using the wild-type homozygotes as the referent group. We also calculated the $p$ for trend across the genotypes in each exposure category to assess likelihood of that exposure category with each additional minor allele. We also conducted this analysis restricted to nonHispanic Caucasians in the control group due to known variation in eye color, hair color, and other phenotypic features across race groups.

Joint effects of IRF4 and sun sensitivity. For each sun sensitivity exposure, we calculated the OR and $95 \%$ CI for NHL using a common referent group and also stratified by IRF4 genotype under the dominant model, combining heterozygotes and homozygous variants. We calculated the $p$-value for interaction for each exposure and IRF4 SNP for NHL risk based on the scored variable for each risk factor and for the genotype. In these calculations, we scored the genotype using a two-level categorization to assess risk for the presence of a variant allele. Statistical significance for interaction was evaluated with the Wald test in models that included a product term for the scored risk factor and the scored genotype. The category corresponding to the lowest level of sun sensitivity or sun exposure was used as the referent group for each exposure. Analyses were conducted using SAS version 9.1 (SAS Institute, Cary, NC).

\section{Results}

Table 1 shows selected characteristics of the NCI-SEER study population. Briefly, the majority of cases and controls were non-Hispanic Caucasians, cases were slightly younger than controls, and there were slightly more men than women in both cases and controls. Cases and controls were distributed roughly evenly into interview groups A and B. The most common NHL subtypes were diffuse large B-cell lymphoma (DLBCL) and follicular lymphoma.

IRF4 and NHL. Fifteen SNPs in the IRF4 gene were evaluated for associations with non-Hodgkin lymphoma (Supplementary Table 1). We observed a significant decrease in risk for NHL with the IRF4 SNP rs12211228 $\left(\mathrm{OR}_{\mathrm{CG}}=0.81,95 \% \mathrm{CI}=0.65-1.00 ; \mathrm{OR}_{\mathrm{CC}}=0.76,95 \%\right.$ $\mathrm{CI}=0.35-1.65 ; p$-trend $=0.04 ;$ Table 2 ). Results were consistent when restricted to non-Hispanic Caucasian subjects. We observed no statistically significant association between the IRF4 SNP rs12203592 and NHL (Table 2). Further, no other IRF4 SNPs showed a statistically significant association with overall NHL (Supplementary Table 1).

Sun sensitivity and NHL. Lighter eye and hair color were both statistically significantly associated with decreased NHL risk (Table 3). The associations were more pronounced for eye color than hair color, and both associations were consistent in analyses restricted to participants with IRF4 genotype data and/or subjects self-reported as nonHispanic Caucasians. Hours in the mid-day sun in the last 10 years was also associated with decreased NHL risk. Other markers of coloring and sun exposure and sensitivity including skin complexion and reaction to first sun of the season were not associated with NHL risk.

IRF4 and sun sensitivity. Table 4 shows associations between the two a priori IRF4 SNPs of interest (rs12203592 and rs12211228) and measures of sun sensitivity and sun exposure among non-Hispanic Caucasian population controls. Presence of the variant allele in the IRF4 SNP rs12203592 was associated with eye color and hair color. The association between hair color and the IRF4 rs12203592 SNP was most pronounced with increasingly light hair color. Compared to dark brown eyes, the magnitude of association was equivalent for all other eye colors which were strongly associated with the IRF4 rs12203592 variant allele. Curiously, the association between IRF4 rs12203592 with hair color and eye color appear to be in opposite directions; this is consistent with the original GWAS results from Han et al. [17] though was not further explored.

The IRF4 rs12211228 SNP that was associated with NHL was not statistically significantly associated with any of the measures of sun sensitivity or sun exposure in this study (Table 4). All results were consistent in analyses 
Table 1 Characteristics of NCI-SEER case-control study participants

\begin{tabular}{|c|c|c|}
\hline Characteristic & $\begin{array}{l}\text { Controls } \\
n(\%)\end{array}$ & $\begin{array}{l}\text { NHL cases } \\
n(\%)\end{array}$ \\
\hline \multicolumn{3}{|l|}{ SEER registry } \\
\hline Detroit & $139(17)$ & $197(20)$ \\
\hline Iowa & $246(30)$ & $301(30)$ \\
\hline Los Angeles & $199(24)$ & $234(24)$ \\
\hline Seattle & $244(29)$ & $258(26)$ \\
\hline \multicolumn{3}{|l|}{ Sex } \\
\hline Male & $443(54)$ & $536(54)$ \\
\hline Female & $385(47)$ & $454(46)$ \\
\hline \multicolumn{3}{|l|}{ Age (years) } \\
\hline$<50$ & $203(25)$ & $277(28)$ \\
\hline $50-59$ & $177(21)$ & $235(24)$ \\
\hline $60-69$ & $285(34)$ & $311(31)$ \\
\hline $70+$ & $163(20)$ & $167(17)$ \\
\hline \multicolumn{3}{|l|}{ Race/Ethnicity } \\
\hline White, non-Hispanic & $646(78)$ & $828(84)$ \\
\hline Black & $112(14)$ & $64(6)$ \\
\hline Asian/other/unknown & $70(8)$ & $98(10)$ \\
\hline \multicolumn{3}{|l|}{ Education } \\
\hline$<12$ years & $77(9)$ & $98(10)$ \\
\hline $12-15$ years & $485(59)$ & $611(62)$ \\
\hline $16+$ years & $266(32)$ & $280(28)$ \\
\hline \multicolumn{3}{|l|}{ NHL histology } \\
\hline DLBCL & - & $294(30)$ \\
\hline Follicular lymphoma & - & $246(25)$ \\
\hline Marginal zone lymphoma & - & $82(8)$ \\
\hline CLL/SLL & - & $101(10)$ \\
\hline Mantle cell lymphoma & - & $40(4)$ \\
\hline Lymphoplasmacytic lymphoma & - & $24(2)$ \\
\hline Burkitt lymphoma & - & $11(1)$ \\
\hline $\begin{array}{l}\text { Mycosis fungoides/Sézary } \\
\text { syndrome }\end{array}$ & - & $18(2)$ \\
\hline Peripheral T-cell lymphoma & - & $41(4)$ \\
\hline NHL, not otherwise specified & - & $133(13)$ \\
\hline \multicolumn{3}{|l|}{ DNA source } \\
\hline Blood & $598(72)$ & $688(69)$ \\
\hline Buccal & $230(28)$ & $302(31)$ \\
\hline \multicolumn{3}{|l|}{ Interview group } \\
\hline Group A* & $461(56)$ & $547(55)$ \\
\hline Group B** & $367(44)$ & $443(45)$ \\
\hline Total participants & 828 & 990 \\
\hline
\end{tabular}

$C L L / S L L$ chronic lymphocytic leukemia/small lymphocytic lymphoma, $D L B C L$ diffuse large B-cell lymphoma, NCI National Cancer Institute, NHL non-Hodgkin lymphoma, SEER surveillance, epidemiology, and end results

* Group A: extended medical history, use of illicit drugs, family medical history in addition to core questions

** Group B: abbreviated medical history, allergies, hobbies, sun exposure (including eye color and skin complexion), phone use, food frequency questionnaire in addition to core questions expanded beyond non-Hispanic Caucasians and adjusted for race/ethnicity.

The remaining IRF4 SNPs evaluated were not found to be statistically significantly associated with measures of sun sensitivity (data not shown).

Joint effects of IRF4 and sun sensitivity in NHL risk. Table 5 shows the joint effects between eye and hair color and NHL risk for the two a priori IRF4 SNP genotypes. We observed no statistically significant $p$-interactions for either the IRF4 rs12203592 or rs12211228 SNP with hair color or eye color. However, the risk estimates for NHL among individuals with lighter eye and hair color appeared more pronounced in those with either variant IRF4 allele than in those with the corresponding common homozygote genotype. This is also observed in stratified analysis (Supplementary Table 2) whereby associations with NHL for eye and hair color appear more pronounced among those with a variant IRF4 SNP. No joint effects with hair or eye color were observed for the remaining thirteen IRF4 SNPs evaluated (data not shown).

\section{Discussion}

The independent associations observed in our data are consistent with those previously published for sun sensitivity and NHL [19], for IRF4 rs12203592 and hair color [17], and for IRF4 rs12211228 and NHL [8], to which our data contributed. Of note, the IRF4 rs12203592 SNP associated with hair color was not associated with NHL, and the IRF4 rs12211228 SNP associated with NHL was not associated with hair color. The two IRF4 SNPs are neither correlated nor in linkage disequilibrium with one another $\left(r^{2}=0.001, \mathrm{D}^{\prime}=0.04\right)$, and accordingly we found no statistically significant $p$-interactions between IRF4 SNPs, measures of sun sensitivity such as hair color, and NHL risk. We do note that although associations between hair color and NHL risk were observed among those with and without variant IRF4 rs12203592 or rs12211228 alleles, the risk estimates were slightly more pronounced among those with either variant IRF4 genotype. We, therefore, cannot discount a possible interrelationship between the IRF4 SNPs with sun sensitivity and NHL risk given our relatively small sample size to evaluate joint effects. We believe that further evaluation, such as in consortial settings where adequate sample sizes are available to assess joint effects, are needed to determine whether these two pathways both function through immune mediation or separately with one through immune mediation and one through pigmentation.

The major strengths of this study include the use of population-based selection for both cases and controls and 


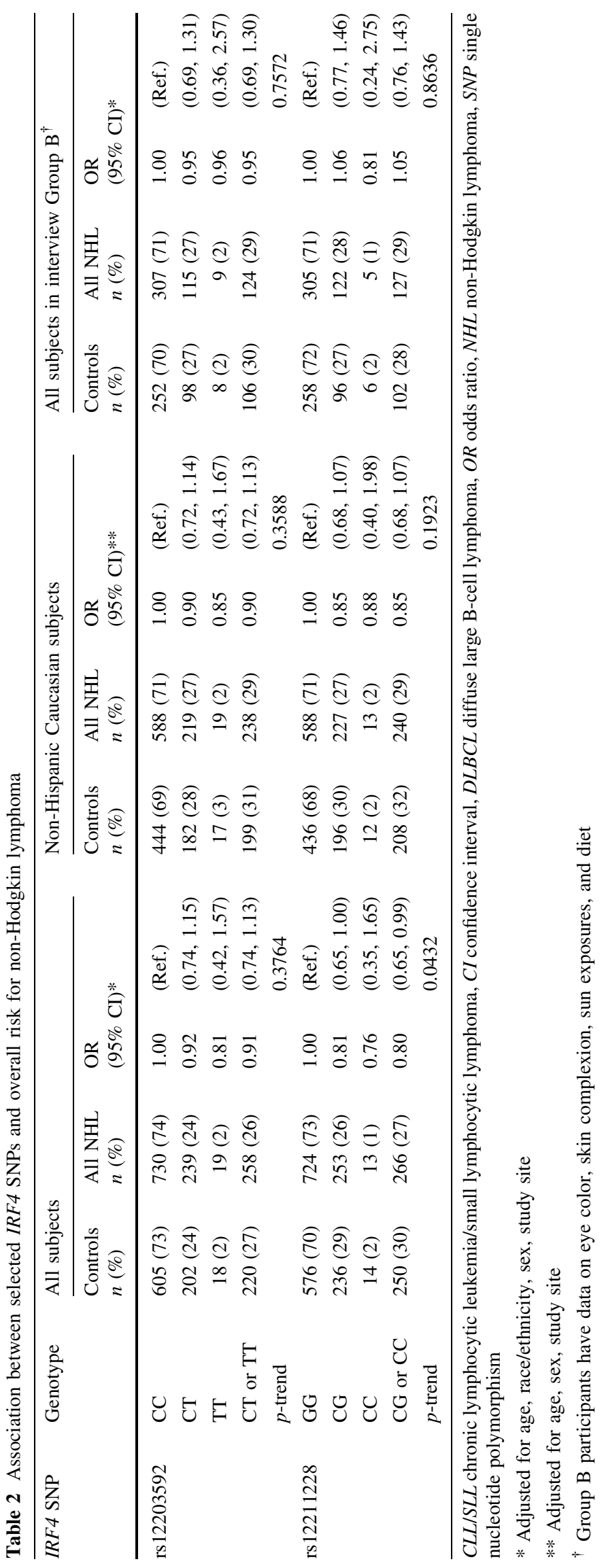




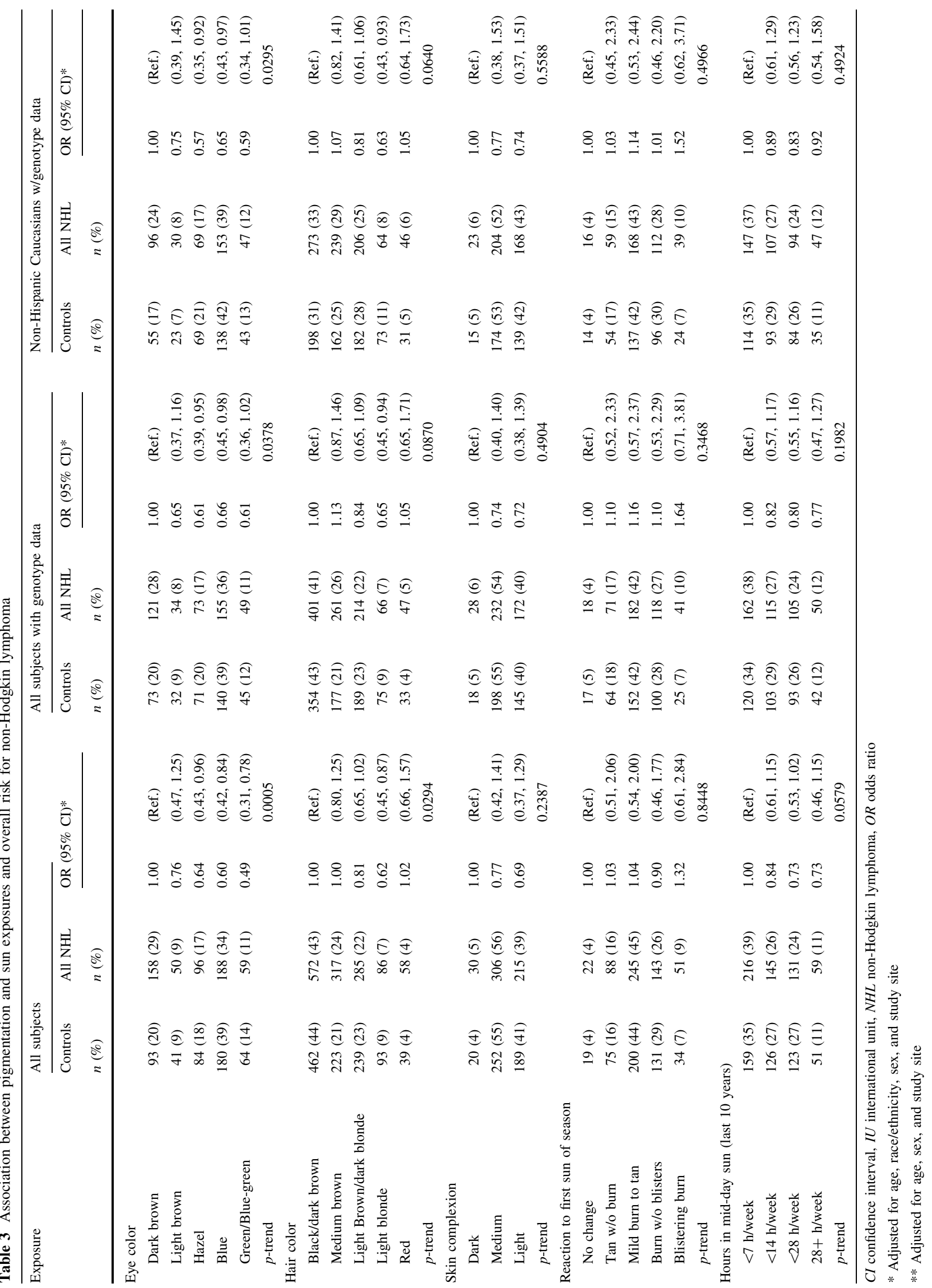




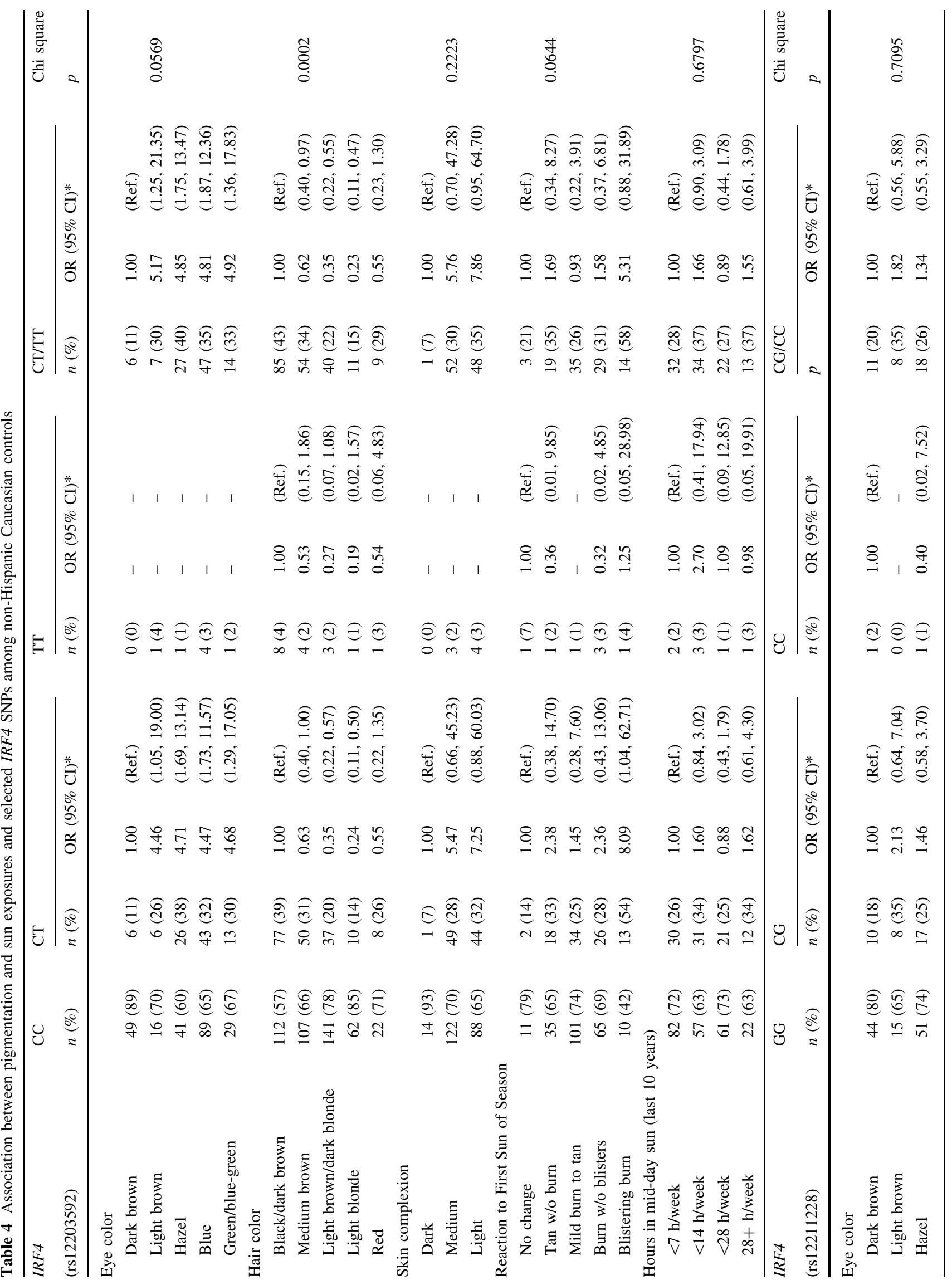




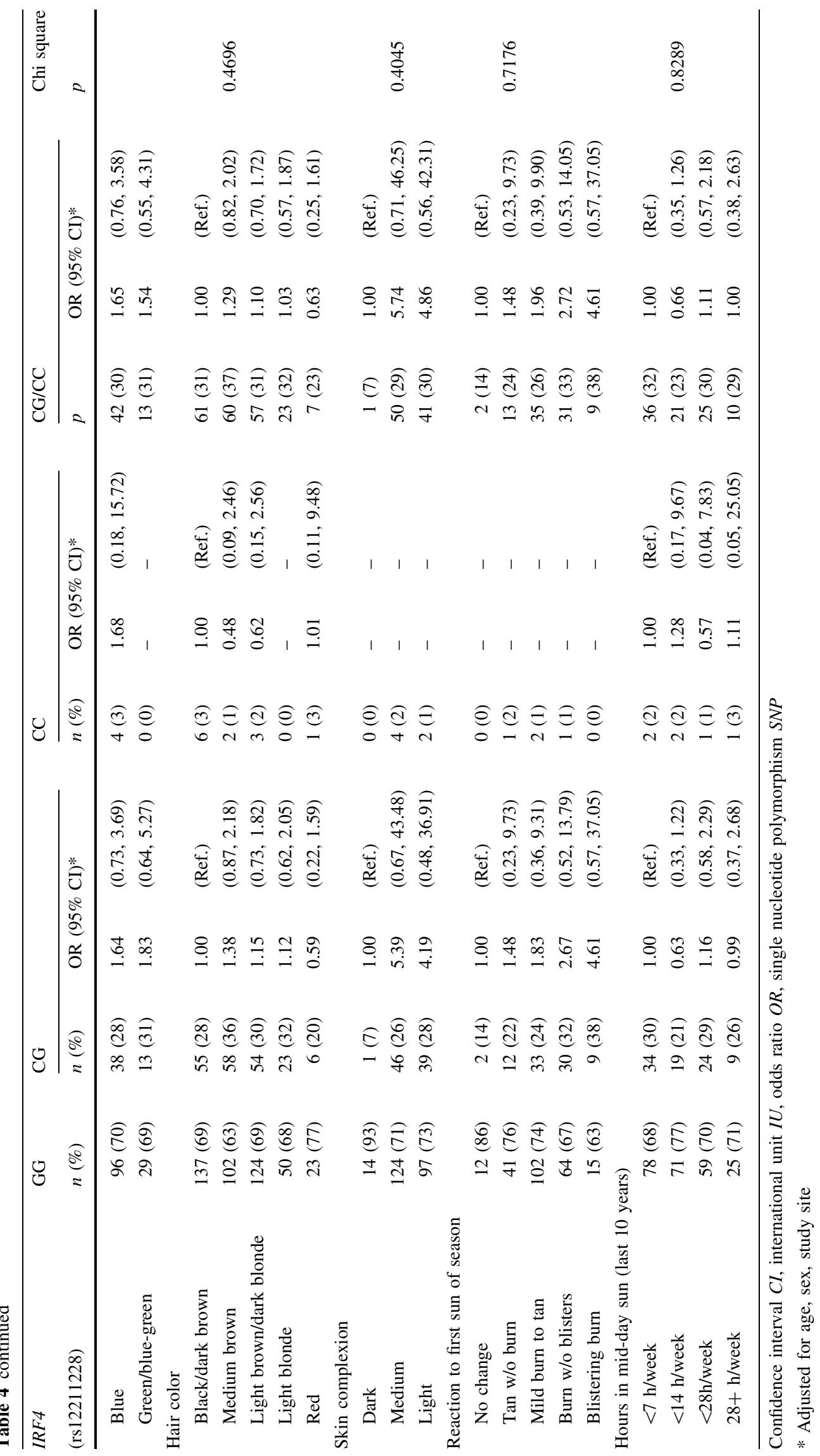


Table 5 Joint effects of eye color and hair color (excluding red hair) and overall non-Hodgkin lymphoma risk with IRF4 genotype, among nonHispanic Caucasians

\begin{tabular}{|c|c|c|c|c|c|c|c|c|}
\hline \multirow{2}{*}{$\begin{array}{l}\text { IRF4 SNP } \\
\text { rs12203592 }\end{array}$} & \multirow{2}{*}{$\begin{array}{l}\text { Exposure } \\
\text { Eye color }\end{array}$} & \multirow[t]{2}{*}{ Genotype } & \multirow{2}{*}{$\begin{array}{l}\text { Controls } \\
n(\%)\end{array}$} & \multirow{2}{*}{$\begin{array}{l}\text { All NHL } \\
n(\%)\end{array}$} & \multicolumn{2}{|c|}{ OR $(95 \% \mathrm{CI})^{*}$} & \multirow[t]{2}{*}{$p$-value } & \multirow[t]{2}{*}{$p$-interaction } \\
\hline & & & & & & & & \\
\hline & Dark brown & $\mathrm{CC}$ & $49(15)$ & $79(20)$ & 1.00 & (Ref.) & & \\
\hline & & $\mathrm{CT} / \mathrm{TT}$ & $6(2)$ & $17(4)$ & 1.66 & $(0.60,4.55)$ & 0.3259 & \\
\hline & Light brown & $\mathrm{CC}$ & $16(5)$ & $20(5)$ & 0.80 & $(0.37,1.71)$ & 0.5589 & \\
\hline & & $\mathrm{CT} / \mathrm{TT}$ & $7(2)$ & $10(3)$ & 0.83 & $(0.29,2.37)$ & 0.7312 & \\
\hline & Hazel & $\mathrm{CC}$ & $41(13)$ & $49(12)$ & 0.74 & $(0.43,1.29)$ & 0.2944 & \\
\hline & & $\mathrm{CT} / \mathrm{TT}$ & $27(8)$ & $20(5)$ & 0.44 & $(0.22,0.88)$ & 0.0204 & \\
\hline & Blue & $\mathrm{CC}$ & $89(27)$ & $99(25)$ & 0.69 & $(0.44,1.11)$ & 0.1241 & \\
\hline & & $\mathrm{CT} / \mathrm{TT}$ & $47(14)$ & $54(14)$ & 0.71 & $(0.41,1.22)$ & 0.2118 & \\
\hline & Green/blue-green & $\mathrm{CC}$ & $29(9)$ & $30(8)$ & 0.58 & $(0.31,1.11)$ & 0.0991 & \\
\hline & & $\mathrm{CT} / \mathrm{TT}$ & $14(4)$ & $16(4)$ & 0.67 & $(0.30,1.52)$ & 0.3356 & \\
\hline & Hair color & & & & & & & 0.6251 \\
\hline & Black/dark brown & $\mathrm{CC}$ & $112(18)$ & $160(21)$ & 1.00 & (Ref.) & & \\
\hline & & $\mathrm{CT} / \mathrm{TT}$ & $85(14)$ & $112(14)$ & 0.92 & $(0.63,1.34)$ & 0.6706 & \\
\hline & Medium brown & $\mathrm{CC}$ & 107 (17) & $166(21)$ & 1.09 & $(0.77,1.54)$ & 0.6402 & \\
\hline & & $\mathrm{CT} / \mathrm{TT}$ & $54(9)$ & $72(9)$ & 0.94 & $(0.61,1.45)$ & 0.7721 & \\
\hline & Light brown/dark blonde & $\mathrm{CC}$ & $141(23)$ & $174(22)$ & 0.85 & $(0.61,1.18)$ & 0.3330 & \\
\hline & & $\mathrm{CT} / \mathrm{TT}$ & $40(7)$ & $32(4)$ & 0.55 & $(0.32,0.94)$ & 0.0282 & \\
\hline & Light blonde & $\mathrm{CC}$ & $62(10)$ & $58(7)$ & 0.65 & $(0.42,1.01)$ & 0.0546 & \\
\hline & & $\mathrm{CT} / \mathrm{TT}$ & $11(2)$ & $6(1)$ & 0.36 & $(0.13,1.00)$ & 0.0496 & \\
\hline \multirow[t]{20}{*}{ rs12211228 } & Eye color & & & & & & & 0.5878 \\
\hline & Dark brown & GG & $44(13)$ & $67(17)$ & 1.00 & (Ref.) & & \\
\hline & & $\mathrm{CG} / \mathrm{CC}$ & $11(3)$ & $29(7)$ & 1.69 & $(0.76,3.77)$ & 0.2011 & \\
\hline & Light brown & GG & $15(5)$ & $23(6)$ & 1.04 & $(0.48,2.25)$ & 0.9121 & \\
\hline & & $\mathrm{CG} / \mathrm{GG}$ & $8(2)$ & $7(2)$ & 0.54 & $(0.18,1.63)$ & 0.2710 & \\
\hline & Hazel & GG & $51(16)$ & $49(12)$ & 0.62 & $(0.36,1.09)$ & 0.0946 & \\
\hline & & $\mathrm{CG} / \mathrm{GG}$ & $18(6)$ & $20(5)$ & 0.73 & $(0.34,1.55)$ & 0.4098 & \\
\hline & Blue & GG & $96(29)$ & $105(27)$ & 0.73 & $(0.45,1.18)$ & 0.1951 & \\
\hline & & $\mathrm{CG} / \mathrm{GG}$ & $42(13)$ & $48(12)$ & 0.76 & $(0.43,1.35)$ & 0.3501 & \\
\hline & Green/blue-green & GG & $29(9)$ & $34(9)$ & 0.71 & $(0.38,1.35)$ & 0.3008 & \\
\hline & & $\mathrm{CG} / \mathrm{GG}$ & $13(4)$ & $13(3)$ & 0.64 & $(0.27,1.52)$ & 0.3096 & \\
\hline & Hair color & & & & & & & 0.3200 \\
\hline & Black/dark brown & GG & $137(22)$ & $187(24)$ & 1.00 & (Ref.) & & \\
\hline & & $\mathrm{CG} / \mathrm{GG}$ & $61(10)$ & $86(11)$ & 1.03 & $(0.69,1.54)$ & 0.8772 & \\
\hline & Medium Brown & GG & $102(17)$ & $176(23)$ & 1.27 & $(0.91,1.78)$ & 0.1550 & \\
\hline & & CG/GG & $60(10)$ & $63(8)$ & 0.76 & $(0.50,1.16)$ & 0.2007 & \\
\hline & Light brown/dark blonde & GG & $124(20)$ & $146(19)$ & 0.85 & $(0.61,1.18)$ & 0.3219 & \\
\hline & & $\mathrm{CG} / \mathrm{GG}$ & $57(9)$ & $60(8)$ & 0.76 & $(0.49,1.17)$ & 0.2066 & \\
\hline & Light blonde & GG & $50(8)$ & $46(6)$ & 0.65 & $(0.41,1.04)$ & 0.0727 & \\
\hline & & $\mathrm{CG} / \mathrm{GG}$ & $23(4)$ & $18(2)$ & 0.58 & $(0.30,1.13)$ & 0.1094 & \\
\hline
\end{tabular}

CI Confidence interval, NHL non-Hodgkin lymphoma, $O R$ odds ratio, SNP single nucleotide polymorphism

* Adjusted for age, sex, and study center

the ascertainment of data on a range of measures designed to assess sun sensitivity and sun exposure in study subjects. Also, our study population reflects all three previously reported independent associations at the basis of this analysis, making it ideal for investigating potential joint effects. Study limitations include lower sample sizes available for some primary and secondary analyses resulting from the split-sample design, which allowed for 
the collection of sun exposure and sun sensitivity data in only half of the participant pool. This decreased the study's power to detect significant interactions. Additionally, the relatively low participation and response rates for the study increase the potential for selection bias in the sample.

We acknowledge that our data may not have detected a joint effect because none existed, because of limited sample size and power to detect a joint effect, or because of our imperfect measures for both genotype and sun exposures, which may have biased our results toward the null. The IRF4 SNPs associated with NHL and hair color were originally identified as part of a tagging algorithm and thus are considered markers of susceptibility. The SNPs evaluated are thus surrogates and likely in linkage disequilibrium with the causal SNP. In addition, the specific mechanism by which sun sensitivity and sun exposure affects NHL risk is unknown and the pigmentation phenotypes such as hair color are considered surrogates for this measure. We, therefore, cannot exclude the possibility that our null observations for joint effects between IRF4 and sun sensitivity are due to our having a poor surrogate marker for the causal factor(s). Finally, we also cannot exclude the possibility that joint effects may exist for specific NHL subtypes which we were unable to evaluate due to small numbers in our study.

In summary, our data support that genetic polymorphisms in the immune regulatory gene IRF4 are linked to both risk for non-Hodgkin lymphoma and to hair color and other phenotypic measures of sun sensitivity and exposure. Further evaluation of joint effects in independent and larger populations is needed. If joint effects are shown, further investigations to identify the mechanisms by which sun sensitivity or exposure and IRF4 genes function to modulate risk for NHL are warranted.

\begin{abstract}
Acknowledgments We gratefully acknowledge the staff and scientists at the SEER centers in Iowa, Los Angeles, Detroit, and Seattle for conducting the study's fieldwork and collection of biological specimens. We also thank Peter Hui of Information Management Services, Inc. for his programming support. This research was supported by the Intramural Research Program (IRP) of the NIH, National Cancer Institute. Financial support was provided by Public Health Service (PHS) contracts: N01-PC-65064, N01-PC-67008, N01-PC-67009, N01-PC-67010, N01-OC-71105.
\end{abstract}

Open Access This article is distributed under the terms of the Creative Commons Attribution Noncommercial License which permits any noncommercial use, distribution, and reproduction in any medium, provided the original author(s) and source are credited.

\section{References}

1. Hartge P, Bracci P, Wang S (2006) Non-Hodgkin lymphoma. In: Schottenfeld D, Fraumeni J Jr (eds) Cancer epidemiology and prevention. Oxford Press, Oxford
2. Chang E, Smedby K, Hjalgrim H et al (2005) Family history of hematopoietic malignancy and risk of lymphoma. J Natl Cancer Inst 97(19):1466-1474

3. Chatterjee N, Hartge P, Cerhan J et al (2004) Risk of nonHodgkin's lymphoma and family history of lymphatic, hematologic, and other cancers. Cancer Epidemiol Biomarkers Prev 13(9):1415-1421

4. Goldgar D, Easton D, Cannon-Albright L, Skolnick M (1994) Systematic population-based assessment of cancer risk in firstdegree relatives of cancer probands. J Natl Cancer Inst 86(21):1600-1608. doi:10.1093/jnci/86.21.1600

5. Wang S, Slager S, Brennan P et al (2007) Family history of hematopoietic malignancies and risk of non-Hodgkin lymphoma (NHL): a pooled analysis of 10211 cases and 11905 controls from the International Lymphoma Epidemiology Consortium (InterLymph). Blood 109(8):3479-3488. doi:10.1182/blood2006-06-031948

6. Rothman N, Skibola C, Wang S et al (2006) Genetic variation in TNF and IL10 and risk of non-Hodgkin lymphoma: a report from the InterLymph Consortium. Lancet Oncol 7(1):27-38. doi: 10.1016/S1470-2045(05)70434-4

7. Skibola C, Curry J, Nieters A (2007) Genetic susceptibility to lymphoma. Haematologica 92(7):960-969. doi:10.3324/ haematol.11011

8. Wang S, Purdue M, Cerhan J et al. (2009) Common genetic variations in the tumor necrosis factor (TNF) and TNF receptor superfamilies and nuclear factor kappa beta transcription factors and risk of non-Hodgkin lymphoma. Plos One (in press)

9. Eisenbeis C, Singh H, Storb U (1995) Pip, a novel IRF family member is a lymphoid-specific, PU.1-dependent transcriptional activator. Genes Dev 9(11):1377-1387. doi:10.1101/gad. 9.11.1377

10. Grossman A, Mittrücker H, Nicholl J et al (1996) Cloning of human lymphocyte-specific interferon regulatory factor (hLSIRF/ hIRF4) and mapping of the gene to 6p23-p25. Genomics 37(2):229-233. doi:10.1006/geno.1996.0547

11. Matsuyama T, Grossman A, Mittrücker H et al (1995) Molecular cloning of LSIRF, a lymphoid-specific member of the interferon regulatory factor family that binds the interferon-stimulated response element (ISRE). Nucleic Acids Res 23(12):2127-2136. doi:10.1093/nar/23.12.2127

12. Yamagata T, Nishida J, Tanaka S et al (1996) A novel interferon regulatory factor family transcription factor, ICSAT/Pip/LSIRF, that negatively regulates the activity of interferon-regulated genes. Mol Cell Biol 16(4):1283-1294

13. Fillatreau S, Radbruch A (2006) IRF4-a factor for class switching and antibody secretion. Nat Immunol 7(7):704-706. doi:10.1038/ni0706-704

14. Klein U, Casola S, Cattoretti G et al (2006) Transcription factor IRF4 controls plasma cell differentiation and class-switch recombination. Nat Immunol 7(7):773-782. doi:10.1038/ni1357

15. Falini B, Fizzotti M, Pucciarini A et al (2000) A monoclonal antibody (MUM1p) detects expression of the MUM1/IRF4 protein in a subset of germinal center B cells, plasma cells, and activated T cells. Blood 95(6):2084-2092

16. Sundram U, Harvell J, Rouse R, Natkunam Y (2003) Expression of the B-cell proliferation marker MUM1 by melanocytic lesions and comparison with S100, gp100 (HMB45), and MelanA. Mod Pathol 16(8):802-810. doi:10.1097/01.MP.0000081726.49886. $\mathrm{CF}$

17. Han J, Kraft P, Nan H et al (2008) A genome-wide association study identifies novel alleles associated with hair color and skin pigmentation. PLoS Genet 4(5):e1000074. doi:10.1371/journal. pgen. 1000074

18. Hartge P, Lim U, Freedman D et al (2006) Ultraviolet radiation, dietary vitamin $\mathrm{D}$, and risk of non-Hodgkin lymphoma (United 
States). Cancer Causes Control 17(8):1045-1052. doi:10.1007/ s10552-006-0040-8

19. Kricker A, Armstrong B, Hughes A et al (2008) Personal sun exposure and risk of non Hodgkin lymphoma: a pooled analysis from the Interlymph Consortium. Int J Cancer 122(1):144-154. doi:10.1002/ijc. 23003

20. Harris N, Jaffe E, Stein H et al (1994) A revised EuropeanAmerican classification of lymphoid neoplasms: a proposal from the International Lymphoma Study Group. Blood 84(5):13611392

21. Jaffe ES, Harris NL, Stein H, Vardiman JW (2001) Pathology and genetics of tumours of haematopoietic and lymphoid tissues. IARC Press, Lyon
22. Wang S, Cerhan J, Hartge P et al (2006) Common genetic variants in proinflammatory and other immunoregulatory genes and risk for non-Hodgkin lymphoma. Cancer Res 66(19):9771-9780. doi:10.1158/0008-5472.CAN-06-0324

23. García-Closas M, Egan K, Abruzzo J et al (2001) Collection of genomic DNA from adults in epidemiological studies by buccal cytobrush and mouthwash. Cancer Epidemiol Biomarkers Prev 10(6):687-696

24. Packer B, Yeager M, Staats B, et al. (2004) SNP500 Cancer: a public resource for sequence validation and assay development for genetic variation in candidate genes. Nucleic Acids Res. 32(Database issue):D528-32. doi:10.1093/nar/gkh005 